\title{
Optimization of flocculation process for cut-stone wastewater : effect of rapid mix parameters
}

\begin{abstract}
Wastewater produced from stone industry of Iran causes serious environmental problems associated with slurry disposal. In traditional method, many ponds are required for clarifying. The use of filtration and addition of chemicals enhance the efficiency of operation as well as saving laborer cost. The polymer bridging mechanism is responsible for flocculation of suspended particles. It was found that the increase of rapid mix velocity reduced the residual turbidity substantially except for the dosage of $0.2 \mathrm{mg}$ of polymer. The addition of polymer was able to enhance flocs strength. For higher dosages of polymer, a direct relationship between increasing rapid mix velocity and reduction of turbidity was observed. The values of aggregation rate constant Kapp were calculated to evaluate the effects of rapid mix velocity and dosage of polymer on residual turbidity. The maximum value of Kapp $=7.2 \times 10-3$ was obtained for the rapid mix velocity $200 \mathrm{rpm}$ and dosage of polymer $1 \mathrm{mg} / \mathrm{l}$.
\end{abstract}

Keyword: Cut-stone wastewater; Flocculation; Rapid mix; Velocity gradient; Optimization 